Revue des patrimoines

\title{
Relevé photogrammétrique d'objets patrimoniaux : un protocole expérimental pour l'optimisation du placement de caméras pour le relevé aérien
}

Photogrammetric recording of heritage objects, an experimental protocol for the best positioning of cameras in aerial surveys

Renato Saleri et Hervé Lequay

\section{(2) OpenEdition}

Édition électronique

URL : http://journals.openedition.org/insitu/21409

DOI : 10.4000/insitu.21409

ISSN : 1630-7305

Éditeur

Ministère de la Culture

Référence électronique

Renato Saleri et Hervé Lequay, « Relevé photogrammétrique d'objets patrimoniaux : un protocole expérimental pour l'optimisation du placement de caméras pour le relevé aérien », In Situ [En ligne], 39 | 2019, mis en ligne le 23 mai 2019, consulté le 11 juillet 2019. URL : http:// journals.openedition.org/insitu/21409; DOI : 10.4000/insitu.21409

Ce document a été généré automatiquement le 11 juillet 2019.

In Situ Revues des patrimoines est mis à disposition selon les termes de la licence Creative Commons Attribution - Pas d'Utilisation Commerciale - Pas de Modification 4.0 International. 


\section{Relevé photogrammétrique d'objets patrimoniaux : un protocole expérimental pour l'optimisation $\mathrm{du}$ placement de caméras pour le relevé aérien}

Photogrammetric recording of heritage objects, an experimental protocol for the best positioning of cameras in aerial surveys

Renato Saleri et Hervé Lequay

\section{Introduction}

1 Bien avant l'avènement des drones, nombre de vecteurs volants libres ou captifs ont permis l'utilisation aérienne de capteurs divers. On pense bien sûr aux dispositifs stratégiques de surveillance durant les conflits et le survol du territoire à des fins de relevé topographique ou cartographique. L'observation scientifique a bien entendu été pionnière en la matière et depuis le début $\mathrm{du} \mathrm{xx}^{\mathrm{e}}$ siècle, la photographie aérienne a connu un essor considérable, notamment dans le domaine de l'étude du patrimoine archéologique, architectural et urbain. Le développement des drones aériens ${ }^{1}$ a facilité la pratique de la prise de vue aérienne «basse hauteur » et a donné naissance à de nouvelles approches métier s'intéressant au suivi, à la conservation et à la diffusion des connaissances accumulées.

Depuis une quinzaine d'années, le laboratoire $\mathrm{MAP}^{2}$ exploite des vecteurs aériens inhabités téléopérés pour l'observation et le relevé d'objets patrimoniaux. Faisant appel aux techniques SfM (Structure from motion) ${ }^{3}$ les plus récentes, nos travaux nous ont permis de numériser avec une grande précision plusieurs sites remarquables en France mais aussi en Italie et en Afrique du Nord notamment. L'expertise développée dans ce domaine 
a fait naturellement converger deux champs disciplinaires dont la technicité et la spécificité apparaissent de plus en plus étroitement liées : toute avancée significative en ce sens mobilise tout autant les connaissances liées à la capture de données (types de caméras, longueurs d'onde, périodicité et étendue des relevés, etc.) que des vecteurs mobilisés pour les obtenir (aérodynamique, protocoles de guidage, stratégies de positionnement, etc.).

Dans le but de comprendre et d'optimiser les campagnes de relevé photographique menant aux processus complexes intervenant durant les phases de calcul algorithmique, nous avons procédé à une série d'expérimentations numériques permettant d'apprécier qualitativement et, dans une moindre mesure, quantitativement, l'impact du positionnement des caméras autour d'un sujet en 3D à restituer. Ce travail de recherche se place dans la perspective des travaux menés dans le cadre du projet Tag3D actuellement en cours de développement. Il s'est déroulé parallèlement à la première phase du projet et a eu comme objectif de consolider les apports de l'équipe M2DisCo du LIRIS $^{4}$ dont les compétences recouvrent à la fois le domaine de l'image numérique et de l'algorithmie géométrique au service de l'optimisation des structures de données, de la mesure de la qualité visuelle et de la modélisation $3 \mathrm{D}^{5}$. Le stage de recherche hébergé au LIRIS a exploré les possibilités d'optimisation du recouvrement photographique d'un objet 3D par une constellation de caméras en exploitant les outils et les ressources logicielles développées par ce laboratoire.

\section{Contexte opérationnel}

4 De nombreux outils de photogrammétrie multi-stéréo (image-based modeling, Structure From Motion algorithms...) sont aujourd'hui disponibles. Depuis les solutions gratuites sur le cloud (Autodesk Recap, 123D catch) aux applications open-source (MicMac, Visual Structure From Motion...) en passant par les outils commerciaux (Pix4D, Photoscan...), ils offrent tous la possibilité de construire rapidement et relativement facilement des géométries 3D sous forme de nuages de points ou de surfaces (meshes) texturées et éventuellement géoréférencées. Les interfaces utilisateur proposent des degrés d'automatisation des processus de saisie différents qui vont du simple "glisser déposer» de photos sur une page web à la saisie de lignes de commandes dans un terminal pour les autres. Il reste que la plupart exploitent les mêmes librairies de calcul, que ce soit pour la recherche automatisée des points homologues inter-images pour la mise en œuvre des routines de calcul des paramètres de calibration internes et externes ou pour la génération de nuages de points denses ou de mailles 3D texturées.

5 La popularité grandissante de ces outils, souvent corrélée à la possibilité d'exporter les modèles générés vers des imprimantes 3D, aujourd'hui de plus en plus abordables, néglige souvent de mentionner les facteurs à prendre en considération afin qu'une campagne photographique soit correctement exploitable. Bien entendu, la même précision ne sera pas requise pour la modélisation d'un objet extrait d'un contexte de loisir que pour la réalisation d'un modèle géométrique qui sera exploité pour des mesures statiques ou des calculs volumétriques. Il est impératif dans ce cas de pouvoir exploiter des campagnes de prises de vues homogènes par leur qualité visuelle (netteté, résolution), optique (ouverture, focale) et computationnelle (discriminance angulaire, taux de recouvrement entre images contiguës...). Il est très difficile d'arbitrer sur le terrain la meilleure stratégie à adopter afin de répondre de manière optimale aux contraintes techniques en 
tenant compte des paramètres propres à la prise de vue (ISO, ouverture, temps de pose...), aux contraintes de recouvrement (auxquelles on répond toujours par «empirisme expérimental ») et aux conditions météo qui peuvent fortement impacter la qualité de la prise de vue voire - le cas échéant - la sécurité des vols en intérieur comme en extérieur (conditions d'éclairage, aérologie...).

6 Dans le domaine du patrimoine, qu'il s'agisse de relevé d'édifices ou d'éléments de décor, il est important - en fonction de la longueur focale utilisée - de déplacer les capteurs «à la bonne distance » des objets dont on effectue le relevé, en tenant compte des impératifs de recouvrement et des effets d'auto-occlusion intervenant immanquablement lors des relevés de décors lapidaires ou statuaires. Lors du « dérushage » des campagnes de prises de vue, on procède d'abord à une sélection minutieuse des clichés afin d'éliminer d'abord ceux qui pourraient venir perturber les passes de calcul (flous de mise au point ou de bougé, contre-jours, reflets excessifs, images sur- ou sous-exposées...). Les images restantes peuvent alors être exploitées numériquement mais très souvent, que ce soit en raison d'un tri trop sélectif ou d'un positionnement lacunaire au plus près d'emplacements difficiles d'accès, les géométries résultantes présentent des «trous » ou des hétérogénéités de densité de maillage ou de texturage. Lorsqu'il est possible de retourner sur le terrain (disponibilité des acteurs concernés, autorisations et démarches administratives...), il sera d'une part pratiquement impossible de retrouver les mêmes conditions d'éclairage et de plus, il y a fort à parier que les éléments de la scène relevée initialement auront changé (véhicules, déplacements inopinés d'éléments du décor...). Dans ces conditions, l'ajout des nouvelles images à la base d'images précédemment utilisées pour la reconstruction risque au mieux de dégrader la qualité des résultats de la campagne, au pire de produire des artefacts gênants.

7 Les modes de vol des drones grand public - extrêmement porteurs du point de vue technologique par leur très forte diffusion commerciale - intègrent des outils permettant aujourd'hui d'effectuer du suivi de cible, par trajectoire linéaire ou circulaire, d'éviter des obstacles et de suivre des trajectoires de vol multi-points suivant le tracé d'un périmètre dessiné à main levée sur une carte géoréférencée. Ils sont de plus capables de revenir à leur point de départ automatiquement si une perte de liaison radio (consignes de vol, télémétrie...) se poursuit au-delà d'une durée déterminée. Les outils professionnels de planification de campagnes de prises de vues aériennes par drone permettent déjà d'optimiser les trajectoires de vol en tenant compte de la focale du capteur, de l'altitude de vol et de la vitesse de translation du vecteur en fonction de la résolution demandée, tout en tenant compte des impératifs de recouvrement longitudinaux et transversaux. Ces paramètres sont "facilement» calculables et développables dans un espace de mesure en 2D, ce qui constitue certainement la majorité des campagnes de prises de vue professionnelles par drone aujourd'hui. En 3D, l'optimisation du placement de réseaux de caméras a fait l'objet de diverses études pour des applications d'inspection 3D et de métrologie ${ }^{6}$ de rendu basé image ${ }^{7}$ ou de surveillance ${ }^{8}$ mais peu d'entre elles se sont intéressées au contexte du patrimoine ou alors, elles se sont limitées à des prises de vue depuis le sol'. Il n'existe pas actuellement, dans le domaine du patrimoine, pour la prise de vue par vecteur aérien autonome, d'outil permettant d'optimiser une campagne de relevé photogrammétrique d'un objet en trois dimensions. Ce cas de figure, qui relève d'un problème NP-difficile, c'est-à-dire pour lequel on ne peut pas trouver de solution exacte rapidement dans tous les $\operatorname{cas}^{10}$, n'a pas véritablement de résolution à ce jour. 
8 La littérature est très fournie en ce qui concerne les espaces de mesure en 2D, notamment en ce qui concerne le relevé « Nadir », c'est-à-dire d'un vol horizontal, capteur embarqué pointant vers le bas pour la cartographie de terrain ou pour l'archéologie. Dans ce contexte, de nombreux sites archéologiques de grande envergure ont été étudiés par le MAP (Pompéi ${ }^{11}$, Vintimille, Saint-Michel-de-Cuxa...). En revanche, on ne peut que constater l'aspect lacunaire de la recherche dans le domaine de l'optimisation du positionnement de capteurs pour le relevé de géométries complexes «en volume $»^{12}$, comme cela pourrait être le cas dans le domaine du relevé patrimonial, aussi bien en extérieur qu'en intérieur, et ceci à toutes les échelles: sont concernés les édifices et les ensembles monumentaux mais également le relevé de sujets peu ou pas déplaçables (éléments de décor, statuaire...).

\section{Le protocole expérimental}

Dans la réalité, on cherche à minimiser les distorsions géométriques du modèle 3D produit en maximisant, là où cela est nécessaire, la densité du nuage de points de la surface 3D résultante et de la texture. En ceci, le placement des caméras lors de l'acquisition est un facteur crucial. Les écarts significatifs dans la précision de la mesure proviennent en général :

10 - d'une distorsion incrémentale apparaissant lors de campagnes de grande dimension effectuées sans l'utilisation de points de repère au sol (Ground Control Points),

11 - d'un facteur de recouvrement inter-images trop faible ou d'une valeur trop importante d'écart angulaire entre elles,

12 - d'une trop grande distance par rapport au sujet relativement à la focale utilisée,

13 - d'une faible granularité de la surface rendant difficile l'identification automatique des points homologues.

14 Parmi ces facteurs, le taux de recouvrement et la distance au sujet nous semblent être les facteurs les plus sensibles au placement des caméras. À cet effet, nous avons modélisé une "caméra virtuelle inverse » à l'aide d'une source lumineuse créée grâce à l'attribut "lumière ponctuelle» dans une scène Autodesk Maya $\odot$. Il existe pour celle-ci une panoplie de modificateurs spécifiques, dont la fonction GOBO qui permet de simuler l'emprise d'un frustum lumineux à l'aide d'un masque texture spécifique. Nous utiliserons également l'attribut d'atténuation linéaire de la luminosité afin d'approcher - comme évoqué ci-dessus - l'effet produit par la distance de la caméra au sujet. Ce modèle très simple rendra possible l'appréciation visuelle et qualitative de l'effet recherché tant que les grandeurs relatives mobilisées dans le modèle resteront homogènes entre elles.

15 Nous avons créé de toutes pièces, à l'aide d'un générateur développé au laboratoire ${ }^{13}$, un modèle architectural expérimental choisi pour ses caractéristiques topologiques contraignantes (développé, masquages, auto-occlusions) et ensuite mis à l'épreuve en exploitant quatre protocoles de placement de caméras parmi les plus utilisés lors des campagnes de relevé: ils sont pour la plupart préconisés par la communauté de chercheurs s'intéressant à ce domaine d'étude ${ }^{14}$ et mis en pratique sur le terrain par les utilisateurs finaux, experts ou non-experts :

16 - campagne circulaire terrestre contre-plongeant,

17 • campagne circulaire aérien oblique horizontal, 
- campagne circulaire aérien oblique plongeant à $45^{\circ}$,

- prise de vue Nadir ${ }^{15}$ en respectant les facteurs de recouvrement intra et inter-bandes le plus communément appliqués (80\% et $60 \%$ ).

Pour cette "caméra virtuelle inverse » qui projettera donc un faisceau lumineux au format des caractéristiques de la caméra embarquée par le drone ayant servi aux séances d'essai - le MAVIC PRO de marque DJI - nous avons retenu les paramètres suivants :

Longueur focale (en $\mathrm{mm}$ ) : 4,73 $\mathrm{mm}$

Taille du pixel (mm) : 0,00161057 x 0,00161057

Taille photosite utile : $4000 \times 3000$ pixels

Field of View (donnée fabricant) : 78, $8^{\circ}$.

Le nombre total de photosites annoncé par le fabricant est de 12,71 Mpix dont une portion est exploitée lors de la prise de vue, le "résiduel périphérique » de 0,71 Mpix agissant comme variable d'ajustement utilisé via la fonction steadyshot intégrée. Le calcul du Field of View se fait par la formule suivante :

ce qui permet de déduire en théorie :

- un FOV horizontal $=2 \cdot \arctan (6,44228 \mathrm{~mm} /(2 \bullet 4.73))=1,196 \mathrm{rad}=\mathbf{6 8}, 56051^{\circ}$

- un FOV vertical $=2 \cdot \arctan (4,83171 \mathrm{~mm} /(2 \bullet 4.73))=0,944 \mathrm{rad}=\mathbf{5 4 , 1 1 4 6 5}$

- un FOV diagonal $=2 \cdot \arctan (8,052849 \mathrm{~mm} /(2 \cdot 4.73))=1,41 \mathrm{rad}=\mathbf{8 0 , 8 2 8 0 2}$

La figure 1 résume l'ensemble des facteurs entrant en compte dans le calcul des paramètres de la caméra (fig. 1).

Figure 1

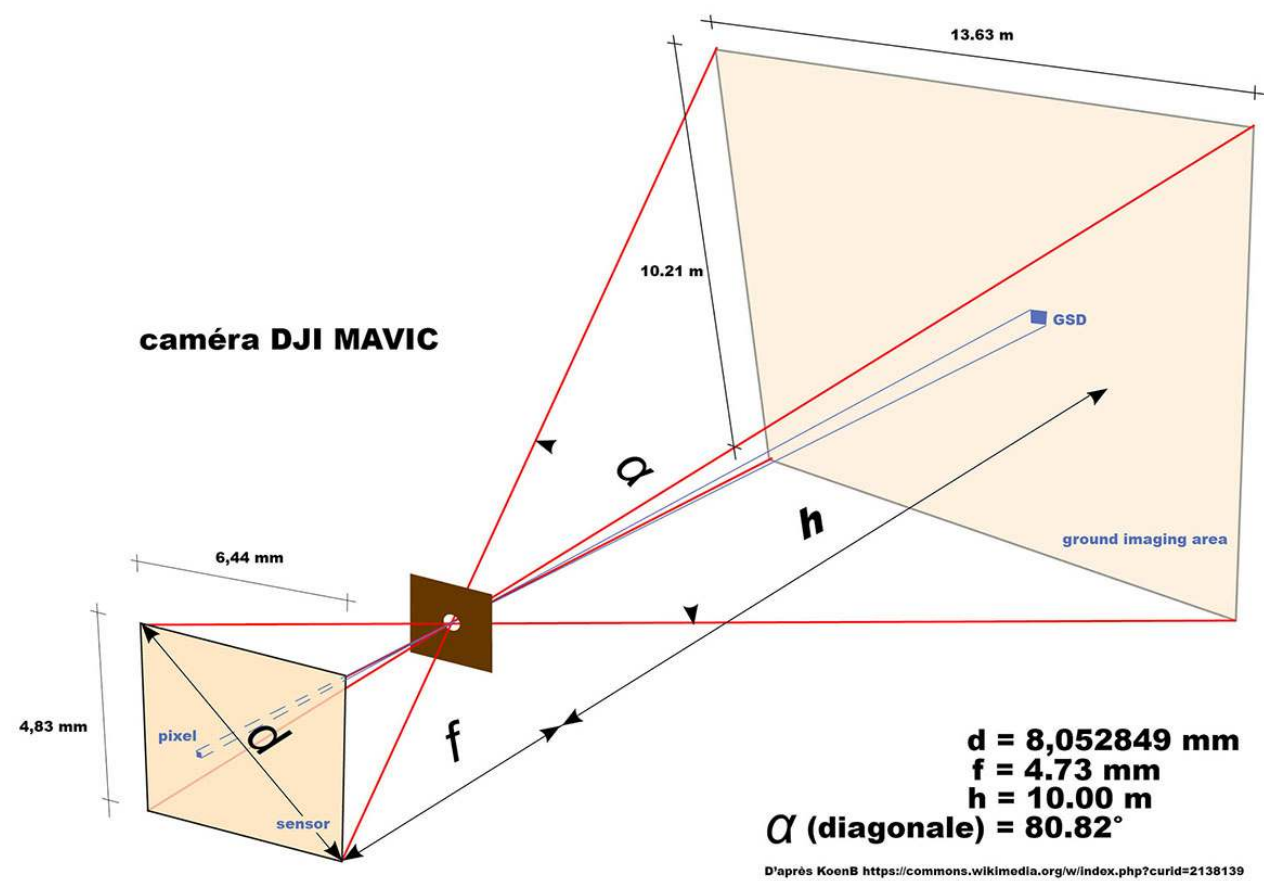

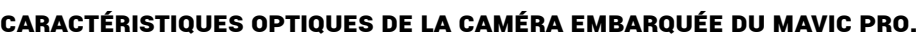

Schéma d'après https://nl.wikipedia.org/wiki/Beeldhoek. 
Ce qui nous permet de déduire l'angle de détection (angle of coverage) théorique en tenant compte des paramètres annoncés par le constructeur, qui sera égal à environ $84,03^{\circ}$. Nous pouvons ainsi calculer la surface couverte en se tenant à $10 \mathrm{~m}$ de distance du sujet en prise de vue Nadir :

- recouvrement horizontal $=\tan (\mathrm{FOV} \mathrm{hz} / 2) \cdot 2=\mathbf{1 3 , 6 3} \mathbf{m}$

- recouvrement vertical $=\tan (\mathrm{FOV} \mathrm{vt} / 2) \cdot 2=\mathbf{1 0 , 2 1} \mathbf{m}$

\section{- GSD théorique $=\mathbf{3 , 4 0 7 5} \mathrm{mm}$}

Ce modèle a été mis à l'épreuve par une mesure réelle du champ de la caméra embarquée: des clichés ont été pris en vol stationnaire au-dessus d'un terrain plat. Quelques mires ont été disposées au sol et déplacées afin d'occuper "visuellement » la totalité de la zone visible sur le retour vidéo de la station-sol. Nous avons ainsi pu vérifier la justesse de l'estimation numérique en réintégrant une image capturée dans un environnement 3D à l'échelle : les valeurs issues de l'expérience permettent cependant d'observer des écarts limités entre le modèle théorique et la mesure de terrain (fig. 2).

\section{Figure 2}

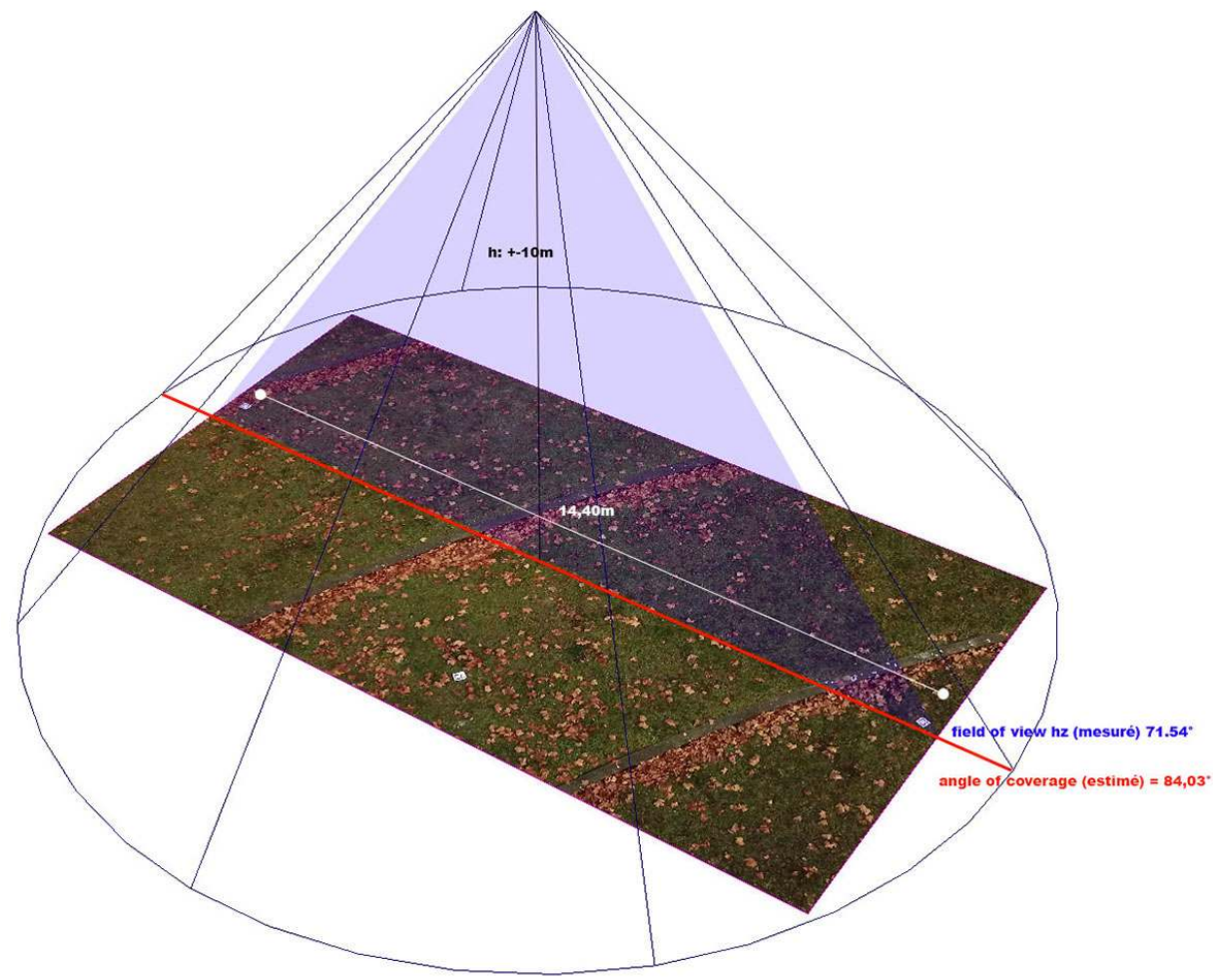

Écarts entre le modèle théorique et la mesure de terrain.

(c) MAP-ARIA.

Nous aurons ainsi :

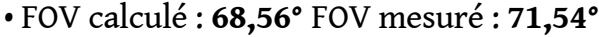

- Champ horizontal calculé : 13,63 m Champ horizontal mesuré : 14,40 m

Remarque : il faut considérer les écarts au regard de la précision instrumentale du baromètre de bord qui place le drone dans une incertitude de positionnement à $+-50 \mathrm{~cm}$ de hauteur. Dans ces 
conditions par exemple, pour un angle donné par le calcul, l'écart d'estimation resterait valide entre 13,25 $\mathrm{m}$ et 14,70 $\mathrm{m}$ pour le champ horizontal.

Nous créons et plaçons ainsi la caméra virtuelle inverse dans Maya en respectant les paramètres retenus pour l'expérience : $10 \mathrm{~m}$ de hauteur avec un angle de détection fixé à $84,03^{\circ}$. Nous choisissons de représenter géométriquement le frustum ainsi créé et de vérifier sa correspondance avec le positionnement du masque-texture appliqué au spot: un rectangle rouge de 13,630 $\mathrm{m} \times 10,210 \mathrm{~m}$ positionné au sol, au centre de la projection lumineuse. La superposition des représentations permettra ainsi de vérifier la concordance des paramètres pris en compte (fig. $\mathbf{3}$ ).

\section{Figure 3}

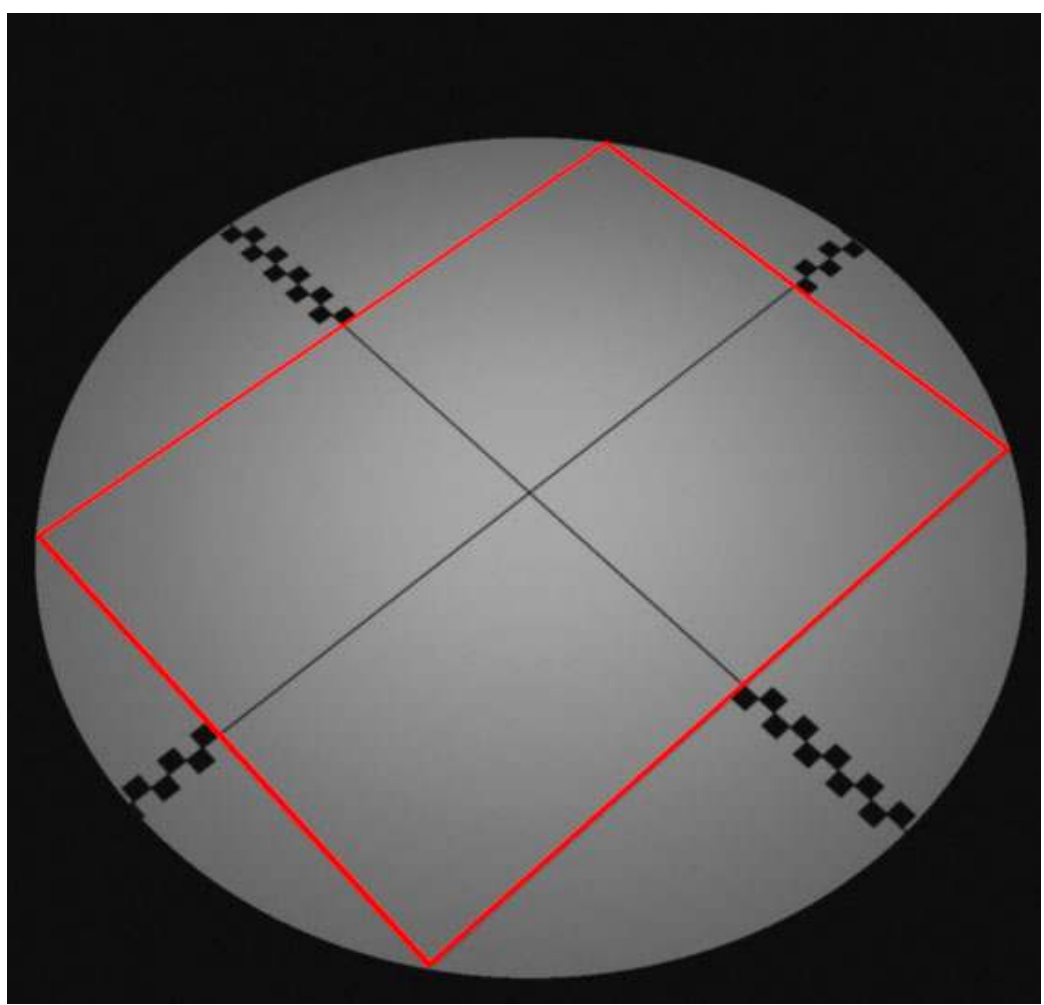

Projection du masque-texture sur la cible de référence.

(c) MAP-ARIA

41 Un premier test consiste à mettre en place 18 "spots-caméras » qui simulent une trajectoire horizontale à $10 \mathrm{~m}$ de hauteur avec une prise de vue nadir; le taux de recouvrement arbitrairement fixé à $80 \%$ inter-images et $60 \%$ intra-images se traduit par un baseline ${ }^{16}$ longitudinal de 2,72 $\mathrm{m}$ et latéral de 2,04 $\mathrm{m}$. Comme prévu, nous observons des zones d'intensité d'éclairage différentes en fonction du nombre de caméras concourantes à cet endroit: la simulation numérique permet d'obtenir une régularité millimétrique dans la répétition des phénomènes, chose que nous n'aurons certainement pas dans la réalité. Nous observons cependant un phénomène résiduel inattendu se produisant en bordure de zone, l'apparition de franges d'intensité cyclique (fig. 4, fig. 5) qui sont fonction d'un pas de prise de vue non synchrone au regard du taux de recouvrement recherché. 
Figure 4

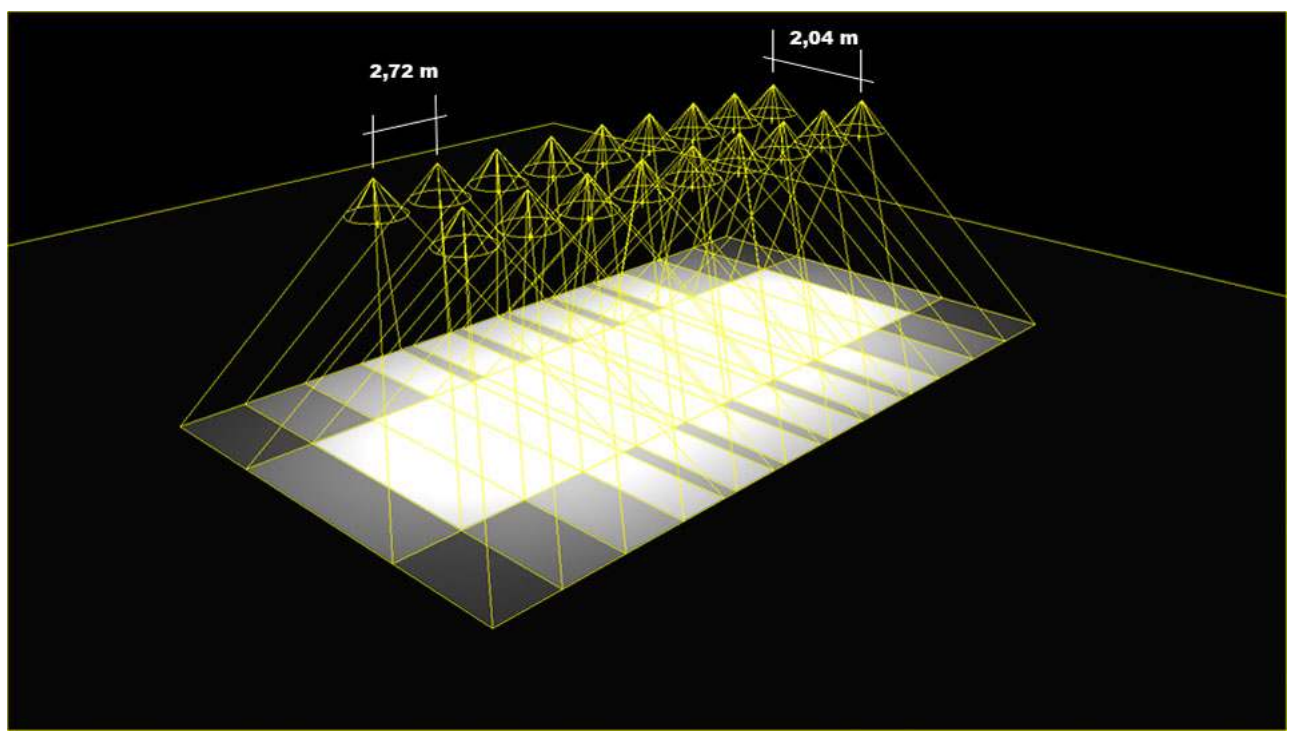

Mise à l'épreuve du modèle dans une scène 3D.

(C) MAP-ARIA

Figure 5

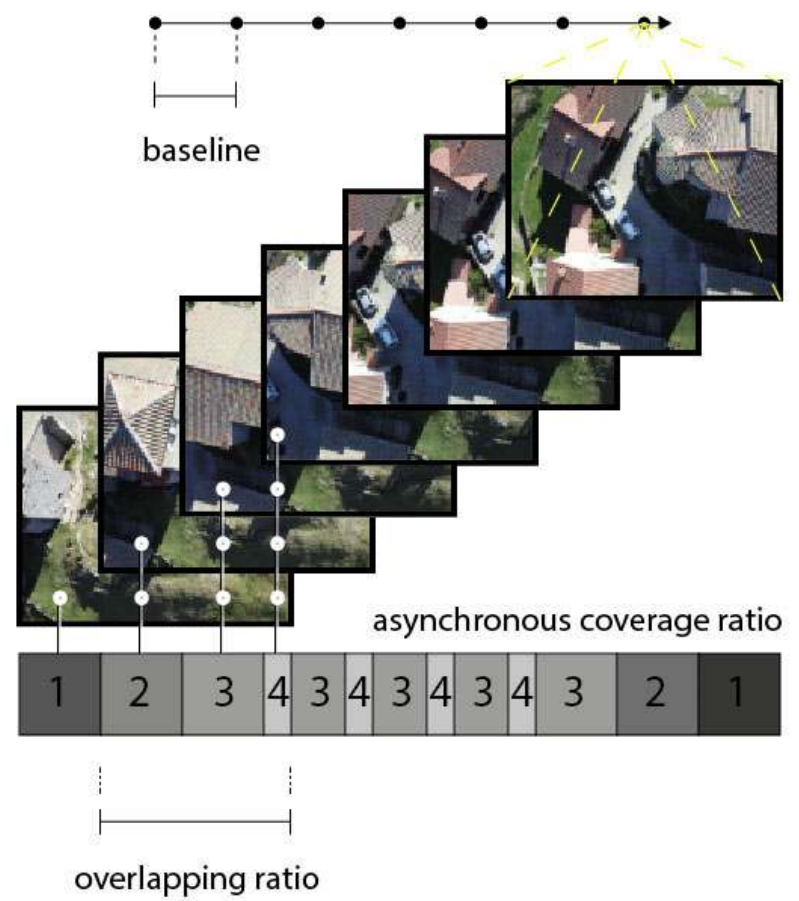

Mise en évidence du caractère asynchrone du recouvrement sur les bords. (C) MAP-ARIA.

Comme évoqué, nous avons réalisé un simulacre pseudo-urbain 3D numérique (fig. 6) à l'aide d'un outil développé au laboratoire. Il est constitué d'un ensemble de 7 « architectones $»^{17}$ de hauteur et de nature différentes qui présente : 
- des hauteurs de bâti différentes

- des toitures plates et en pente

- des volumes imbriqués présentant des redents et des auto-occlusions.

Figure 6

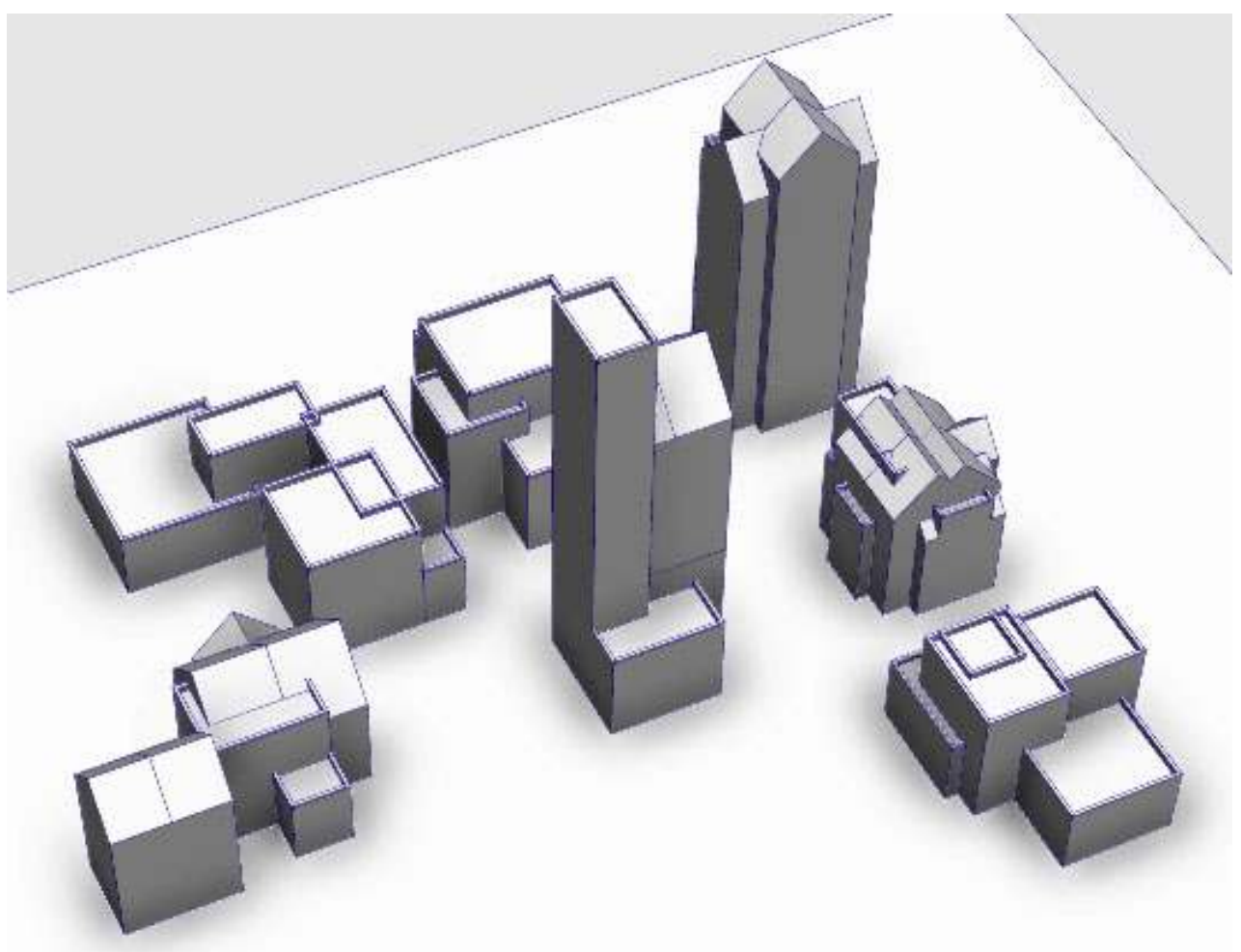

Modèle géométrique utilisé pour l'expérience.

(c) MAP-ARIA.

Sa géométrie est constituée d'un seul bloc avec le socle et a fait l'objet d'un texturage unique par un UVset de 2048 x 2048 pixels enregistré au format TIFF. Nous verserons les résultat du calcul dans le même UVset pour chacune des quatre solutions afin de pouvoir comparer la quantité et la répartition des pixels clairs à la surface de l'image générée par «baking de textures» avec le moteur de rendu Mental Ray® de Maya (voir fig. 9). Bien entendu, pour chaque solution le même nombre de "caméras virtuelles inverses » sera mobilisé, ce qui rendra quantifiable dans une certaine mesure la dispersion de la lumière produite par chaque set de placement de caméras. Nous avons conscience que s'agissant d'un modèle non HDR, nous obtenons un écrêtage des valeurs d'intensité maximale (blanc pur) ; pour une plus grande amplitude de la mesure on pourra utiliser d'autres modes de codage des informations d'image. Une première passe de calculs fait apparaître, comme on pouvait s'y attendre, une nette disparité de l'éclairage des scènes ; nous avons mis en place dans un premier temps quatre capteurs d'éclairage ponctuels sur quatre surfaces distinctes (fig. 7) : le mur d'un bâtiment au centre de la scène (1), le mur d'un bâtiment en périphérie (2), la toiture-terrasse d'un immeuble du centre de la scène (3), le pan d'une toiture inclinée en périphérie (4). Nous allons mesurer la valeur moyenne du niveau de gris de chaque échantillon prélevé au droit de la zone correspondante dans la mappe UV générée. Le choix de ces quatre typologies de placement nous permettra déjà de caractériser l'efficacité de la couverture d'éléments distincts du point de vue 
architectural : maçonneries verticales, toitures plates ou inclinées. L'observation de ces indicateurs sera corroborée par un calcul de l'aire de l'histogramme, plus pertinent selon nous quant à la caractérisation de l'efficacité de l'éclairage global mais moins discriminant quant à l'identification des éléments architecturaux potentiellement impactés par les différents protocoles de placement de caméras.

Figure 7
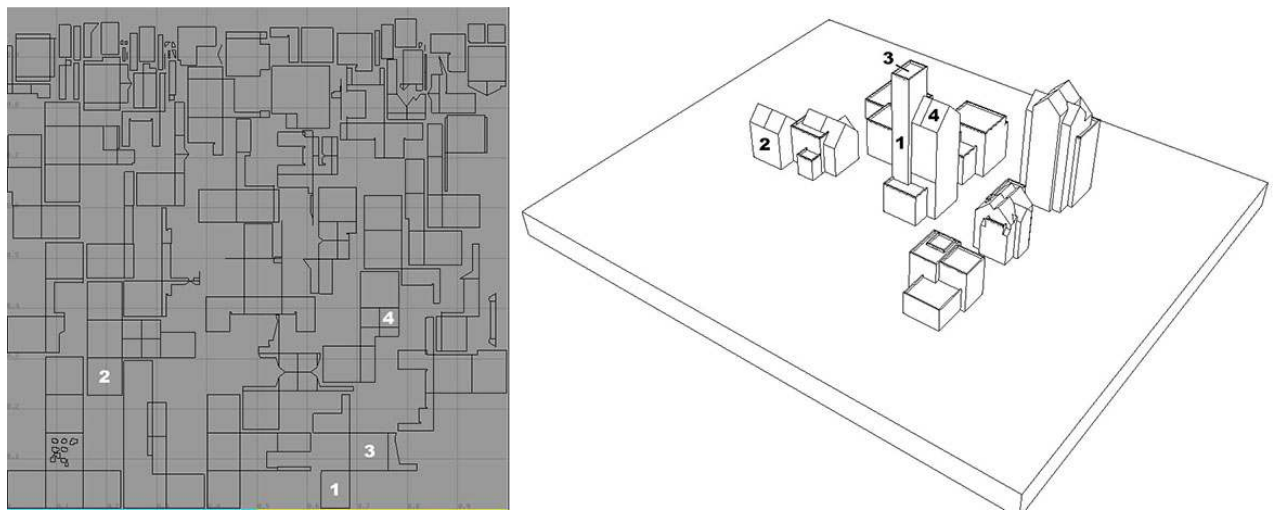

Choix et positionnement des zones tests sur la carte UV.

(c) MAP-ARIA.

\section{Interprétation des données}

47 Si nous exprimons les valeurs de gris ${ }^{18}$ de chaque zone mesurée, nous observons les valeurs suivantes:

\begin{tabular}{|l|l|l|l|l|}
\hline & terrestre & aérien HZ & aérien $45^{\circ}$ & Nadir \\
\hline secteur 1 & 105 & 120 & 116 & 50 \\
\hline secteur 2 & 110 & 130 & 113 & 40 \\
\hline secteur 3 & 0 & 0 & 105 & 73 \\
\hline secteur 4 & 49 & 70 & 116 & 66 \\
\hline Total & 264 & 320 & 450 & 229 \\
\hline Moyenne & 66 & 80 & 112,5 & 57,25 \\
\hline Écart-type & 110 & 50 & 11 & 33 \\
\hline
\end{tabular}

Il est intéressant de remarquer la valeur totale qui correspond à une quantité de lumière ayant frappé simultanément les quatre surfaces placées différemment dans la scène : une valeur importante tend à indiquer une couverture plus importante par les caméras ou un placement «au plus près» du sujet, alors que la faible valeur de l'écart type semble 
représenter une plus grande homogénéité et une moindre dispersion de la distribution de lumière. Les prises de vue circulaires horizontales ou terrestres favorisent légèrement la définition des éléments verticaux, surtout en périphérie si l'on considère le rayon du cercle de prise de vue constant. Les prises de vues plongeantes éclairent davantage en revanche les toitures en pente, probablement par un placement localement frontal lors du passage des caméras à proximité. La meilleure qualité de la prise de vue plongeante par rapport à une prise de vue nadir sur des toitures plates est probablement dû à une multiplication des prises de vues concourantes lors des vols circulaires, surtout sur les éléments de plus grande hauteur. Les campagnes basse hauteur et à plus forte raison, terrestres, étant - comme on peut l'imaginer et au vu des valeurs du tableau - totalement inefficaces pour le relevé des éléments de toiture horizontaux et très faiblement opérationnels pour les pans de toiture inclinés.

Un premier rendu dans Maya, le texture UV placement étant identique pour les quatre rendus (fig. 8, fig. 9) :

Figure 8

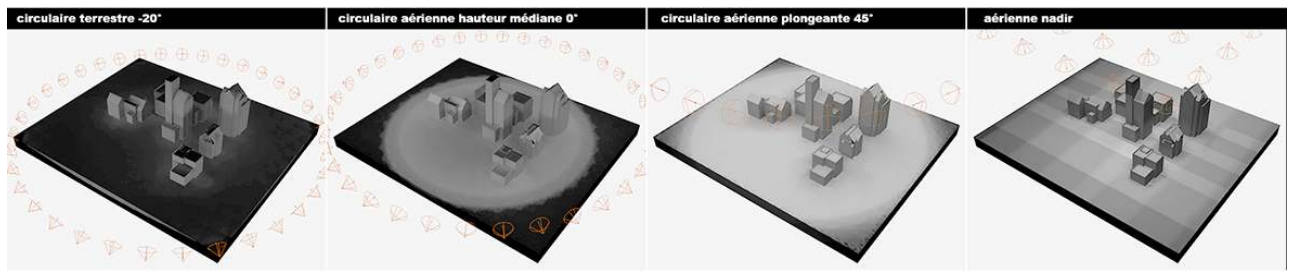

Rendu des quatre modèles d'éclairage.

(C) MAP-ARIA.

Figure 9

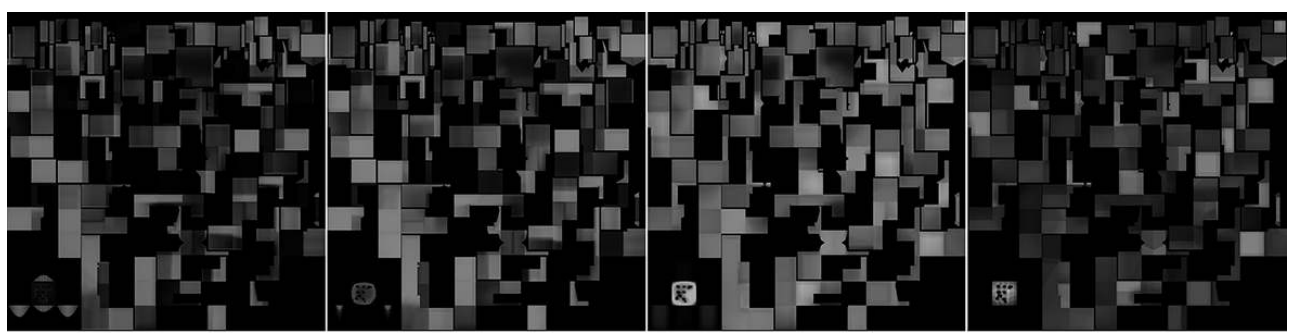

Stockage des valeurs d'éclairage sur les cartes UV respectives.

(c) MAP-ARIA.

La hauteur de la caméra au moment de la prise de vue impacte logiquement la concentration de la lumière et la localisation des disparités d'éclairage (voir fig. 8, fig. 9) . Afin de mettre en évidence ces phénomènes d'accumulation, nous avons procédé à un filtrage du fichier texture par un calcul d'isohélie ${ }^{19}$ sur quatre niveaux (fig. 10). Nous avons ensuite généré les histogrammes correspondants, sur lesquels d'ultérieures observations ont été effectuées: bien entendu, un décalage de l'histogramme vers la droite caractérise une meilleure valeur globale d'éclairement (surexposition) mais lors du filtrage de l'image avec une fonction isohélie, nous pouvons observer une modification très nette des valeurs moyennes et médianes respectives. Il reste à valider l'hypothèse selon laquelle ces indicateurs statistiques pourraient également fournir un indicateur convergeant à la caractérisation d'une «meilleure » trajectoire de vol. 
Figure 10

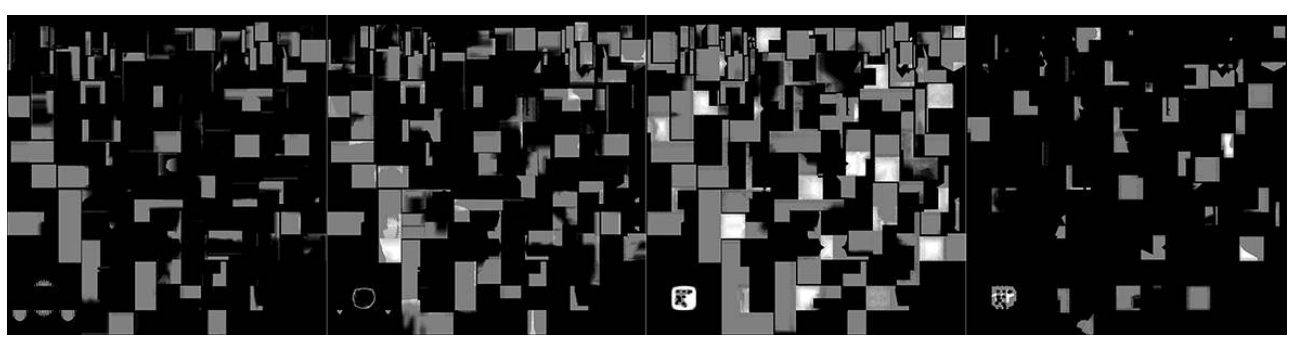

Application d'un isohélie de quatre niveaux sur les cartes UV d'origine.

(c) MAP-ARIA.

La plupart des phénomènes révélés répondent logiquement au protocole de placement mobilisé, mais il y en a de plus inattendus comme ces zones d'occlusion à la base des bâtiments en vue plongeante à $45^{\circ}$ (2) ainsi que la plus forte concentration de lumière annulaire lors d'une prise de vue aérienne à l'horizontale, ceci étant probablement dû à des phénomènes de masquage de la scène par les bâtiments adjacents lorsque la caméra se situe à faible hauteur (fig. 11, fig. 12). La prise de vue nadir produit - et c'est plus inattendu - une beaucoup plus faible couverture relative des éléments verticaux (4) nonobstant la courte focale de la caméra embarquée. Nous remarquons en revanche une hétérogénéité significative dans l'intensité de l'éclairement entre les toitures horizontales et les toitures inclinées en mode plongeant à $45^{\circ}(3)$, alors que cet effet est plus homogène dans la prise de vue nadir (voir tableau ci-dessus).

Figure 11

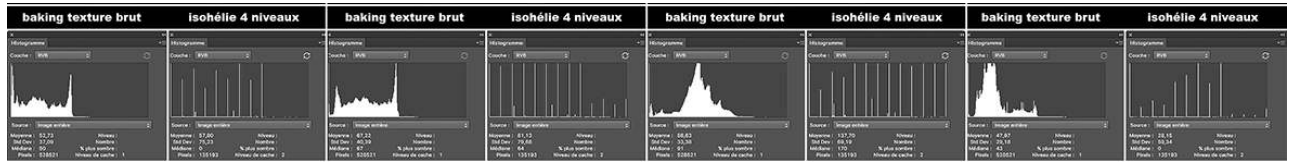

Les histogrammes comparés des cartes UV pour chacune des trajectoires de vol.

(c) MAP-ARIA.

Figure 12

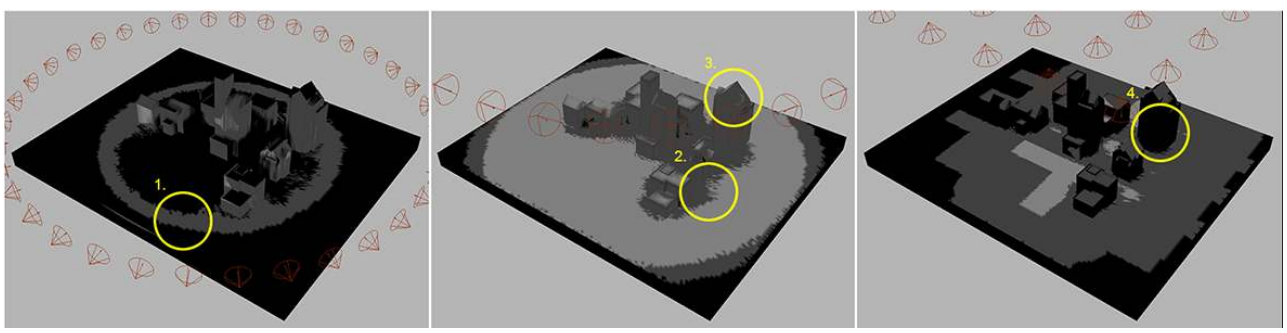

Les zones d'intérêt mises en évidence par un filtrage par isohélie de la carte UV.

(c) MAP-ARIA.

\section{Attentes et perspectives}

Le travail présenté, qui s'inscrit dans un projet de recherche en cours, vise à optimiser le positionnement de caméras autour d'objets patrimoniaux pour produire des objets 3D par 
photogrammétrie multi-stéréo. Ce problème est - nous l'avons vu - NP-difficile et il n'existe pas à ce jour de stratégie opératoire optimale pour décider a priori qu'une campagne de prise de vue répondra mieux qu'une autre aux besoins opérationnels d'un traitement avec les outils SfM existants. La recherche menée a donc pour but de mettre en place un protocole rapide d'évaluation des trajectoires possibles qui puissent " envelopper " au mieux l'objet à relever en minimisant le temps de vol, toujours coûteux en termes d'énergie et de sécurité. Cette première phase de l'étude consiste donc à objectiver l'efficacité d'un recouvrement quelconque, compte tenu de la quantité de lumière distribuée sur une surface déployée en 2D. Dans la réalité, il présuppose l'existence d'un modèle 3D préliminaire grossier qui peut être rapidement obtenu par une première passe de prise de vues aériennes "classique»: une campagne circulaire plongeante à $45^{\circ}$ par exemple. Nous imaginons à ce stade faire appel à des solutions logicielles éprouvées permettant d'arbitrer l'efficacité de solutions calculées en les faisant converger vers des optimums pondérés: nous pourrions par exemple chercher à maximiser la quantité de pixels clairs répartis sur une surface développée tout en minimisant le nombre de sources de lumière utilisées pour produire cet éclairement ou à réduire au maximum le chemin reliant les caméras. Nous avons vu que des transformations d'une carte UV permettent de mettre en évidence certains indicateurs qui pourraient se révéler discriminants quant à l'arbitrage objectif de solutions optimales. Il est possible également d'améliorer localement la précision du modèle géométrique : il faudra à cet effet multiplier les stations à proximité du détail, rapprocher si possible la caméra du sujet ou utiliser de plus longues focales, ce qui peut être pénalisant pendant les phases de corrélation et produire des images bruitées ou floues. Nous imaginons à cet effet explorer plus avant les technologies SLAM ou Next Best View pour lesquelles un état de l'art détaillé est en cours d'élaboration. Cette approche pourrait être exploitée en complément de la méthode détaillée ci-dessus afin d'améliorer localement et «à la demande » la précision du modèle 3D au fur et à mesure de la progression de la campagne photographique. Les méthodes et les outils afférents au relevé patrimonial s'adossent aujourd'hui fortement aux recherches effectuées dans le domaine de la vision par ordinateur, de l'intelligence artificielle ou de la robotique embarquée. Ces technologies se fraient lentement un chemin vers les environnements logiciels grand public dédiés aux supports hypermédias les plus récents (télés 3D, casques immersifs ou dispositifs de réalité augmentée...) et se diffuseront de plus en plus au sein des communautés s'intéressant au patrimoine. Mais c'est grâce aux contributions des architectes, historiens ou archéologues fortement impliqués dans la recherche et le développement d'outils et de contenus que le potentiel de ces technologies innovantes trouvera un essor pertinent pour la constitution de connaissances nouvelles sur le patrimoine et de leur transmission auprès du plus grand nombre. 


\section{BIBLIOGRAPHIE}

ABERGEL, Violette, SALERI, Renato et LEQUAY, Hervé, 2017. « Vecteurs aériens téléopérés pour l'acquisition de données spatiales d'objets patrimoniaux, retour d'expérience ». Revue française de photogrammétrie et de télédétection, 2017, no 213-214.

ALSADIK, Bashar, GERKE, Markus et VOSSELMAN, George. « Automated camera network design for 3D modeling of cultural heritage objects ». Journal of Cultural Heritage, novembre 2013, vol. 14, $\mathrm{n}^{\circ}$ 6. DOI 10.1016/j.culher.2012.11.007.

BODOR, Robert, DRENNER, Andrew, SCHRATER, Paul et PAPANIKOLOPOULOS, Nikolaos. « Optimal Camera Placement for Automated Surveillance Tasks ». Journal of Intelligent and Robotic Systems, 12 octobre 2007, vol. 50, n 3. DOI 10.1007/s10846-007-9164-7.

CLÉRY, Isabelle et PIERROT-DESSEILLIGNY, Marc. « An ergonomic interface to compute 3D models using photogrammetry ». Dans Proceedings du XXIII ${ }^{e}$ symposium de la CIPA. Prag. S.l. : s.n., 2011

FLEISHMAN, Shachar, COHEN-OR, Daniel et LISCHINSKI, Dani. « Automatic Camera Placement for Image-Based Modeling ». Dans Computer Graphics Forum. S.l. : Wiley Online Library, 2000.

GEORGANTAS, Athanasios, BRÉDIF, Mathieu et PIERROT-DESSEILLIGNY, Marc. « An accuracy assessment of automated photogrammetric techniques for 3D modelling of complex interiors ». International Archives of the Photogrammetry, Remote Sensing and Spatial Information Sciences, 2012, vol. 39 .

MAVRINAC, Aaron. Modeling and optimizing the coverage of multi-camera systems. Thèse, Electrical and Computer Engineering. Université de Windsor (Ontario, Canada), 2012.

MEGUERDICHIAN, Seapahn, SLIJEPCEVIC, Sasa, KARAYAN, Vahag et POTKONJAK, Miodrag. «Localized algorithms in wireless ad-hoc networks: location discovery and sensor exposure ". Dans Proceedings of the 2nd ACM international symposium on Mobile ad hoc networking \& computing. S.l. : ACM, 2001.

NOCERINO, Erica, MENNA, Fabio, REMONDINO, Fabio et SALERI, Renato. « Accuracy and block deformation analysis in automatic UAV and terrestrial photogrammetry-Lesson learnt ». ISPRS. Annals of the Photogrammetry, Remote Sensing and Spatial Information Sciences, 2013, vol. 2, n 5/W1.

OLAGUE, Gustavo. Planification du placement de caméras pour des mesures 3D de précision. Thèse de doctorat. Grenoble : Institut national polytechnique de Grenoble, 1998.

O'ROURKE, Joseph. « Open problem from art gallery solved ». Int. Journal of Computational Geometry and Applications, 1992, vol. 2.

SALERI, Renato. "Pseudo-Urban automatic pattern génération ». International Nonlinear Sciences Conference 2003 [en ligne]. S.l. : s.n. février 2003. Voir le site : https://halshs.archives-ouvertes.fr/ halshs-00262448 [consulté le 15/02/2018].

SALERI, Renato. "Génération automatique de morphologies architecturales et urbaines ». Dans Architectures, Urbanisme et Géométries [en ligne]. S.l. : s.n., octobre 2004. Voir le site : https:// halshs.archives-ouvertes.fr/halshs-00271458 [Consulté le 15/02/2018]. 
SALERI, Renato, CAPPELLINI, Valeria, NONY, Nicolas, DE LUCA, Livio, PIERROT-DESEILLIGNY, Marc, BARDIÈRE, Emmanuel et CAMPI, Massimiliano. « UAV photogrammetry for archaeological survey: The Theaters area of Pompeii ». Dans : [en ligne] S.l. : IEEE, octobre 2013. Voir le site : http://ieeexplore.ieee.org/document/6744818/ [consulté le 15/02/ 2018].

URIBE LOBELLO, Ricardo, DUPONT, Florent et DENIS, Florence. « Out-of-core adaptive iso-surface extraction from binary volume data ». Graphical Models, novembre 2014, vol. 76, nº 6. DOI 10.1016/ j.gmod.2014.06.001.

VIDAL, Vincent, WOLF, Christian et DUPONT, Florent. « Combinatorial mesh optimization ». The Visual Computer, 2012, vol. 28, $\mathrm{n}^{\circ} 5$.

VIDAL, Vincent, WOLF, Christian et DUPONT, Florent. « Mesh Segmentation and Global 3D Model Extraction ». Dans Symposium on Geometry Processing. S.l. : s.n., 2012.

ZHANG, Xuebo, ALARCON-HERRERA, Jose Luis et CHEN, Xiang. « Optimization for 3D model-based multi-camera deployment ». Dans IFAC Proceedings Volumes, 2014, vol. 47, $\mathrm{n}^{\circ} 3$.

\section{NOTES}

1. - ABERGEL, Violette, SALERI, Renato et LEQUAY, Hervé. «Vecteurs aériens téléopérés pour l'acquisition de données spatiales d'objets patrimoniaux, retour d'expérience ». Revue française de photogrammétrie et de télédétection, 2017, $\mathrm{n}^{\circ}$ 213-214, p. 73-79.

2. - UMR 3495 CNRS / Ministère de la Culture.

3. - NOCERINO, Erica, MENNA, Fabio, REMONDINO, Fabio et SALERI, Renato. « Accuracy and block deformation analysis in automatic UAV and terrestrial photogrammetry-Lesson learnt ». ISPRS. Annals of the Photogrammetry, Remote Sensing and Spatial Information Sciences, 2013, vol. 2, n 5/W1, p. 203-208.

4. - UMR 5205 CNRS / INSA Lyon / Université Claude Bernard-Lyon-1 / Université LumièreLyon-2 / École Centrale de Lyon.

5. - URIBE LOBELLO, Ricardo, DUPONT, Florent et DENIS, Florence. «Out-of-core adaptive isosurface extraction from binary volume data ». Graphical Models, novembre 2014, vol. 76, $\mathrm{n}^{\circ} 6$, p. 593-608. DOI 10.1016/j.gmod.2014.06.001; VIDAL, Vincent, WOLF, Christian et DUPONT, Florent. "Mesh Segmentation and Global 3D Model Extraction». Dans Symposium on Geometry Processing. S.l.: s.n., 2012, p. inconnue; Ibid. "Combinatorial mesh optimization ». The Visual Computer, 2012, vol. 28, n 5, p. 511-525.

6. - OLAGUE, Gustavo. Planification du placement de caméras pour des mesures 3D de précision. Thèse de doctorat. Grenoble: Institut national polytechnique de Grenoble, 1998 ; MAVRINAC, Aaron. Modeling and optimizing the coverage of multi-camera systems. Thèse, Electrical and Computer Engineering. Université de Windsor (Ontario, Canada), 2012; ZHANG, Xuebo, ALARCONHERRERA, Jose Luis et CHEN, Xiang. «Optimization for 3D model-based multi-camera deployment ». Dans IFAC Proceedings Volumes, 2014, vol. 47, n³, p. 10126-10131.

7. - FLEISHMAN, Shachar, COHEN-OR, Daniel et LISCHINSKI, Dani. « Automatic Camera Placement for Image-Based Modeling». Dans Computer Graphics Forum. S.l. : Wiley Online Library, 2000, p. $101-110$.

8. - BODOR, Robert, DRENNER, Andrew, SCHRATER, Paul et PAPANIKOLOPOULOS, Nikolaos. «Optimal Camera Placement for Automated Surveillance Tasks ». Journal of Intelligent and Robotic Systems, 12 octobre 2007, vol. 50, nº 3, p. 257-295. DOI 10.1007/s10846-007-9164-7.

9. - ALSADIK, Bashar, GERKE, Markus et VOSSELMAN, George. «Automated camera network design for 3D modeling of cultural heritage objects ». Journal of Cultural Heritage, novembre 2013, vol. 14, n 6, p. 515-526. DOI 10.1016/j.culher.2012.11.007. 
10. - MEGUERDICHIAN, Seapahn, SLIJEPCEVIC, Sasa, KARAYAN, Vahag et POTKONJAK, Miodrag. «Localized algorithms in wireless ad-hoc networks: location discovery and sensor exposure ». Dans Proceedings of the 2nd ACM international symposium on Mobile ad hoc networking \& computing. S.l. : ACM, 2001, p. 106-116.

11. - SALERI, Renato, CAPPELLINI, Valeria, NONY, Nicolas, DE LUCA, Livio, PIERROT-DESEILLIGNY, Marc, BARDIÈRE, Emmanuel et CAMPI, Massimiliano. «UAV photogrammetry for archaeological survey: The Theaters area of Pompeii ». Dans : [en ligne] S.l. : IEEE, octobre 2013, p. 497-502. Voir le site: http://ieeexplore.ieee.org/document/6744818/ [consulté le 15/02/2018]; NOCERINO, Erica, MENNA, Fabio, REMONDINO, Fabio et SALERI, Renato. Art. cit.

12. - O'ROURKE, Joseph. "Open problem from art gallery solved». Int. Journal of Computational Geometry and Applications, 1992, vol. 2, p. 215-217.

13. - SALERI, Renato. "Pseudo-Urban automatic pattern génération». International Nonlinear Sciences Conference 2003 [en ligne]. S.l. : s.n. février 2003. Voir le site: https://halshs.archivesouvertes.fr/halshs-00262448 [consulté le 15/02/2018]; Id. "Génération automatique de morphologies architecturales et urbaines ». Dans Architectures, Urbanisme et Géométries [en ligne]. S.1.: s.n. octobre 2004. Voir le site: https://halshs.archives-ouvertes.fr/halshs-00271458 [consulté le 15/02/2018].

14. - CLÉRY, Isabelle et PIERROT-DESSEILLIGNY, Marc. «An ergonomic interface to compute 3D models using photogrammetry ». Dans Proceedings du XXIII ${ }^{e}$ symposium de la CIPA. Prag. S.l. : s.n., 2011 ; GEORGANTAS, Athanasios, BRÉDIF, Mathieu et PIERROT-DESSEILLIGNY, Marc. "An accuracy assessment of automated photogrammetric techniques for 3D modelling of complex interiors ». International Archives of the Photogrammetry, Remote Sensing and Spatial Information Sciences, 2012, vol. 39, p. 23-28.

15. - Direction située selon la verticale et vers le centre de la Terre. Il est à l'opposé du Zénith.

16. - Distance intra et inter-bandes entre les caméras.

17. - SALERI, Renato. «Pseudo-Urban automatic pattern génération ». Art. cit. ; Id. «Génération automatique de morphologies architecturales et urbaines ». Art. cit.

18. - Les valeurs de gris étant stockées sur 8 bits peuvent représenter une variation d'intensité allant de 0 à 255.

19. - Dans les logiciels de traitement d'image, l'outil isohélie permet de déterminer le nombre de plages de couleur à conserver et arrondit ainsi la valeur de chaque pixel à la valeur d'isohélie la plus proche.

\section{RÉSUMÉS}

Les drones professionnels sont devenus les compagnons indispensables des campagnes de relevé photogrammétrique et LIDAR (light détection and ranging); dans ce domaine, les pratiques métier du secteur agricole ou industriel sont aujourd'hui largement consolidées : elles mettent en œuvre des protocoles extrêmement rodés en ce qui concerne l'optimisation des trajectoires de vol pour la mesure ou la surveillance du territoire. Dans ce contexte, ces pratiques s'affranchissent assez facilement des contraintes de positionnement optimal dans la mesure où il s'agit la plupart du temps de répéter des vols identiques au-dessus de sujets de nature similaire : carrières, étendues agricoles, lignes électriques, infrastructures routières ou ferroviaires... Dans le domaine du patrimoine, l'hétérogénéité du corpus d'étude soumet les opérateurs à des arbitrages 
opérationnels empiriques, qui seront fonction de la nature, de l'étendue et de la topologie des objets à mesurer : il n'existe pas aujourd'hui de procédure permettant de planifier a priori le positionnement de caméras - a fortiori d'un drone - autour d'un objet patrimonial « en trois dimensions ». L'expérience des utilisateurs, amateurs éclairés ou professionnels, sera meilleure juge dans la plupart des cas. Le MAP collabore actuellement avec une équipe du laboratoire LIRIS (Laboratoire d'informatique en image et systèmes d'information) à un défi interdisciplinaire IMAG'IN cofinancé par le CNRS : il conjugue ces empirismes expérimentaux testés depuis près de 15 ans par le laboratoire dans le domaine de la photogrammétrie aérienne avec les avancées scientifiques pour les mettre au service de la compréhension géométrique et topologique des sujets d'étude, afin de réaliser un outil d'assistance "de terrain» capable de guider les opérateurs vers les choix opérationnels - terrestres ou aériens - les plus pertinents lors des campagnes de relevé. Dans cet article, nous présenterons une méthode expérimentée dans le cadre d'une collaboration en cours avec l'équipe M2DisCo (Modèles multirésolution, discrets et combinatoires) du Liris.

Professional drones have become indispensable tools for photogrammetric surveys and light detection and ranging. The trade practices of drone management, applied in agricultural or industrial uses, are now largely established, implementing well-rehearsed protocols for the optimisation of flight patterns for the measurement or surveillance of a given territory. In this context, the practices do not necessarily involve the best possible positioning of cameras since, more often than not, it is a question of repeating identical flight patterns over subjects of a broadly similar nature: quarries, agricultural surfaces, overhead power lines, road or railway infrastructures... Where the heritage is concerned, however, the heterogeneous nature of the objects observed means that drone operators have to make empirical operational choices according to the nature, the scale and the topology of the object being recorded or measured. Today, there are no preliminary procedures that allow for planning the best camera positions around a three-dimensional heritage object, particularly if these cameras are attached to drones. The experience of the users, whether they are professionals or enlightened amateurs, is usually the best judge here. The MAP is collaborating today with a team from the LIRIS laboratory (Laboratoire d'informatique en image et systèmes d'information) to work on an interdisciplinary IMAG'IN project, co-financed by the CNRS. The project is based on the experimental and empirical approaches developed over the last fifteen years by the laboratory in the field of aerial photogrammetry, combined with scientific advances. The aim is to facilitate the geometrical and topological understanding of the objects studied in order to offer a 'field' tool of assistance, guiding operators towards the most pertinent operational choices, on the ground or in the air, for surveying campaigns. This article presents a method tried out in the framework of an ongoing collaboration between the M2DisCo team (Modèles multirésolution, discrets et combinatoires) and Liris.

\section{INDEX}

Mots-clés : acquisition de données spatiales, procédures SfM, modélisation géométrique 3D, drones, automatisation du placement de caméras

Keywords : spatial data capture, SfM procedures, 3D geometric modelling, drones, automatization of camera placing 


\section{AUTEURS}

RENATO SALERI

Architecte DPLG, responsable de la plate-forme drone MAP et ENSAL, MAP-ARIA, UMR 3495

CNRS/MCC renato.saleri@lyon.archi.fr

HERVÉ LEQUAY

Architecte DPLG, maître de conférence des ENSA, responsable scientifique de l'équipe MAP-aria, MAP-ARIA, UMR 3495 CNRS/MCC herve.lequay@lyon.archi.fr 